\section{The Implications of the Introduction of Neoadjuvant Chemoradiotherapy for Oesophageal Cancer in a Low Volume Centre}

\section{Abstract}

Background: Oesophagectomy is the standard treatment for oesophageal cancer in medically fit patients, but is associated with significant morbidity and mortality. Neoadjuvant chemoradiotherapy has been included in the management of this disease with the aim to improve prognosis, and has been shown to improve survival without an associated increase in operative complications. This study included patients who underwent oesophagectomy at the Canberra Hospital, to determine if neoadjuvant therapy was associated with an increase in perioperative morbidity and mortality.

Methods: Patients who underwent oesophagectomy at Canberra Hospital between January 2001 and December 2012 were reviewed retrospectively. Statistical analysis was performed using Chi-Squared tests, time to disease recurrence was estimated using Kaplan-Meier methods.

Results: Of 98 patients ( 73 men, 25 women; median age 63 years) who underwent oesophagectomy, 39 (40\%) received neoadjuvant therapy. Perioperative complications of grades III (67\%) and IV (100\%), occurred in the surgery alone group. Perioperative mortality rate was $3.8 \%$, with all deaths occurring in the surgery alone group $(\mathrm{P}=0.05)$. Complete resection was possible in 87 patients $(89 \%), 100 \%$ in the neoadjuvant therapy group, $82 \%$ in the surgery alone group $(P=0.10)$. Median disease free survival was 49.2 months $(\mathrm{Cl} 25.2,73.2)$, with no significant difference between patient groups $(P=0.53)$.

Neoadjuvant therapy was associated with lower perioperative morbidity, with no statistically significant increase in perioperative mortality. It was also associated with improved rates of complete resection; however this did not appear to have translated into improved disease free survival.

Keywords: Oesophageal Cancer; Neoadjuvant Chemotherapy; Adenocarcinoma; Oesophagectomy

\author{
Hazel Serrao-Brown* and \\ James Fergusson \\ Department of Surgery, Canberra Hospital, \\ Yamba Drive, Garran ACT 2605, Australia
}

Corresponding author:

Hazel Serrao-Brown

Đ hazel.g.serrao-brown@act.gov.au

Department of Surgery, Canberra Hospital, Yamba Drive, Garran ACT 2605, Australia.

Tel: (02)62442222

Citation: Serrao-Brown H, Fergusson J (2018) The Implications of the Introduction of Neoadjuvant Chemoradiotherapy for Oesophageal Cancer in a Low Volume Centre. J Univer Surg. Vol.6 No.1:9

Received: February 10, 2018; Accepted: February 13, 2018; Published: February 15, 2018

\section{Introduction}

Oesophageal cancer remains the seventh leading cause of cancer related mortality in the world [1], and the majority of patients present at an advanced stage of disease [2,3] Oesophagectomy was the standard treatment for localised oesophageal cancer, and remains a high risk procedure associated with significant morbidity and mortality $[1,4,5]$. In order to improve outcomes, the management of this disease has evolved to become tri- modal, combining neoadjuvant chemotherapy and radiotherapy with surgical resection $[1,6]$.

A multimodal treatment approach for oesophageal cancer could involve neoadjuvant chemotherapy, along with radiotherapy [6]. Neoadjuvant chemotherapy may have several advantages, such as the facilitation of complete resection by tumour downstaging $[6,7]$ improved tolerance when compared to postoperative chemotherapy [6] and the early treatment of micrometastases $[6,7]$. Similarly, neoadjuvant radiotherapy has been shown to 
improve the pathological complete response rate and local tumour downstaging [6,8]. However, the disadvantages of neoadjuvant therapy may include delayed surgery, impaired wound healing, increased risk of respiratory complications as well as increased morbidity and mortality of the oesophagectomy itself $[6,8]$. The CROSS trial demonstrated improved rates of both overall and disease-free survival among patients who received neoadjuvant therapy followed by surgery $[6,8,9]$. Moreover, Hagry et al. [10] Concluded that neoadjuvant therapy has acceptable complication rates relative to its effectiveness in down-staging of tumours, resulting in an improved chance for complete resection [10]. This study aims to identify the effect of neoadjuvant therapy on perioperative morbidity and mortality, by performing a retrospective review of patients who underwent oesophagectomy for oesophageal cancer at Canberra Hospital.

\section{Methods}

The medical records for all patients who underwent an oesophagectomy for oesophageal cancer at the Canberra Hospital, from $1^{\text {st }}$ January 2001 through $31^{\text {st }}$ December 2012, were reviewed. Ninety-eight patients were identified, all of whom had histologically confirmed primary squamous cell carcinoma, adenocarcinoma or adenosquamous carcinoma of the oesophagus or gastro-oesophageal junction. Patients with cancer found to be arising from the stomach were excluded from this review. Patients with stage IV cancer did not undergo oesophagectomy, and were excluded from this study.

Patients were placed in one of two groups based on whether they received neoadjuvant therapy or not, as prescribed by medical and radiation oncologists. For each group, mean age, gender, post-operative histology tumour site, tumour type and tumour stage based on the American Joint Committee for Cancer Staging ( $5^{\text {th }}$ edition), were reviewed and recorded into a database (Table 1).

The majority of patients who underwent neoadjuvant therapy were treated with a standard protocol of cisplatin and infusional 5-fluorouracil, and 45 Gy external beam radiation. Oesophagectomy was performed using the Ivor-Lewis procedure. Histologically confirmed complete tumour resection was defined as R0, and incomplete resection was defined as R1, where tumour was histologically found to extend to within $1 \mathrm{~mm}$ of the surgical margin. Perioperative morbidity and mortality were classified according to the Clavien-Dindo classification of surgical complications, occurring within 30 days.10 Patients were followed up to monitor for recurrence by reviewing medical records up to and including 31 $1^{\text {st }}$ December 2014.

Data were analysed using SPSS, version 20. The following endpoints were analysed using Chi Squared tests: perioperative complication grade, disease recurrence and complete resection rate. Disease free survival, comparing each group, was estimated using Kaplan-Meier methods.

This study was approved by the Human Research Ethics Committee, ACT Government Health Directorate.

\section{Results}

Ninety-eight patients (73 men and 25 women, median age 63.4 years) with cancer of the oesophagus or gastrooesophageal junction were reviewed, all of whom underwent oesophagectomy between January, 2001 and December, 2012. Of these patients, 39 underwent neoadjuvant therapy, while 59 underwent surgery alone. Of the 39 patients that underwent neoadjuvant therapy, 26 (67\%) received chemotherapy alone, 3 $(8 \%)$ received radiotherapy alone, while $10(26 \%)$ received both. The majority of tumours were adenocarcinomas (83\%) located in the oesophagus (81\%). The majority of patients had stage III oesophageal cancer (51\%). The median age of patients receiving neoadjuvant therapy was 61 years, compared to 64 years in the surgery alone group (Table 1 ). The percentage of patients who underwent neoadjuvant therapy increased markedly for the years 2007-2012 when compared to the years 2001-2006 (Table 2).

Perioperative complications were recorded as grades I to V, most of the patients with grade III and all patients with complications of grade IV occurred in the group that did not receive neoadjuvant therapy (Table 3). The perioperative mortality rate was $3.8 \%$ (3 patients), with all deaths (grade $\mathrm{V}$ complication) occurring in the surgery alone group.

R0 resection was achieved in 87 patients (88.8\%). A higher percentage of patients (100\%) who received neoadjuvant therapy had complete resection, compared to those who did not $(81.5 \%)$, although this difference did not reach statistical significance (Table 4)

Table 1 Demographic and clinical characteristics.

\begin{tabular}{|c|c|c|c|}
\hline \multicolumn{4}{|c|}{ Neoadjuvant therapy } \\
\hline & Yes & No & P-Value \\
\hline $\operatorname{Sex}(M / F)$ & $16 / 96$ & $40 / 14$ & 0.255 \\
\hline Mean age ( Years) & 61.08 & 64.46 & 0.198 \\
\hline \multicolumn{4}{|c|}{ Tumour Site } \\
\hline Oesophagus & 20 & 43 & \multirow[b]{2}{*}{0.611} \\
\hline Gastro-oesophageal Junction & 5 & 11 & \\
\hline \multicolumn{4}{|c|}{ Tumour Type } \\
\hline Adenocarcinoma & 20 & 43 & \multirow{3}{*}{1} \\
\hline Adenosquamous Carcinoma & 1 & 1 & \\
\hline Squamous cell carcinoma & 4 & 10 & \\
\hline \multicolumn{4}{|c|}{ AJCC Stage } \\
\hline 0 & 2 & 1 & \multirow{4}{*}{0.025} \\
\hline 1 & 0 & 14 & \\
\hline II & 8 & 12 & \\
\hline III & 15 & 27 & \\
\hline
\end{tabular}

Table 2 The number and proportion of oesophagectomy patients who underwent neoadjuvant therapy for the years 2001-2006 and 2007-2012.

\begin{tabular}{|c|c|c|c|c|}
\hline Year & \multicolumn{2}{c|}{ Neoadjuvant Therapy } & Total & P-Value \\
\hline & Yes & No & & \\
\hline $2001-2006$ & $6(14 \%)$ & $37(86 \%)$ & $43(100 \%)$ & $<0.001$ \\
\hline $2007-2012$ & $33(60 \%)$ & $22(40 \%)$ & $55(100 \%)$ & \\
\hline
\end{tabular}


Table 3: Perioperative complication grade in accordance with the Clavien-Dindo classification of surgical complications.

\begin{tabular}{|c|c|c|c|c|}
\hline $\begin{array}{c}\text { Complication } \\
\text { Grade }\end{array}$ & \multicolumn{2}{|c|}{ Neoadjuvant therapy } & Total & P-value \\
\hline Yes & $15(38 \%)$ & No & & \\
\hline I & $8(21 \%)$ & $7(12 \%)$ & 15 & 46 \\
\hline II & $14(36 \%)$ & $10(17 \%)$ & 24 & \\
\hline III & $2(5 \%)$ & $4(7 \%)$ & 6 & 0.05 \\
\hline IV & $0(0 \%)$ & $4(7 \%)$ & 4 & \\
\hline V & $0(0 \%)$ & $3(5 \%)$ & 3 & \\
\hline Total & 39 & 59 & 98 & \\
\hline
\end{tabular}

Table 4 Rates of complete (R0) and incomplete (R1) resection.

\begin{tabular}{|c|c|c|c|c|}
\hline Resection & \multicolumn{2}{|c|}{ Neoadjuvant therapy } & Total & P-value \\
\hline R0 & Yes & No & & \\
\hline R1 & $39(100 \%)$ & $48(82 \%)$ & 87 & \\
\hline Total & $0(0 \%)$ & $11(18 \%)$ & 11 & 0.014 \\
\hline
\end{tabular}

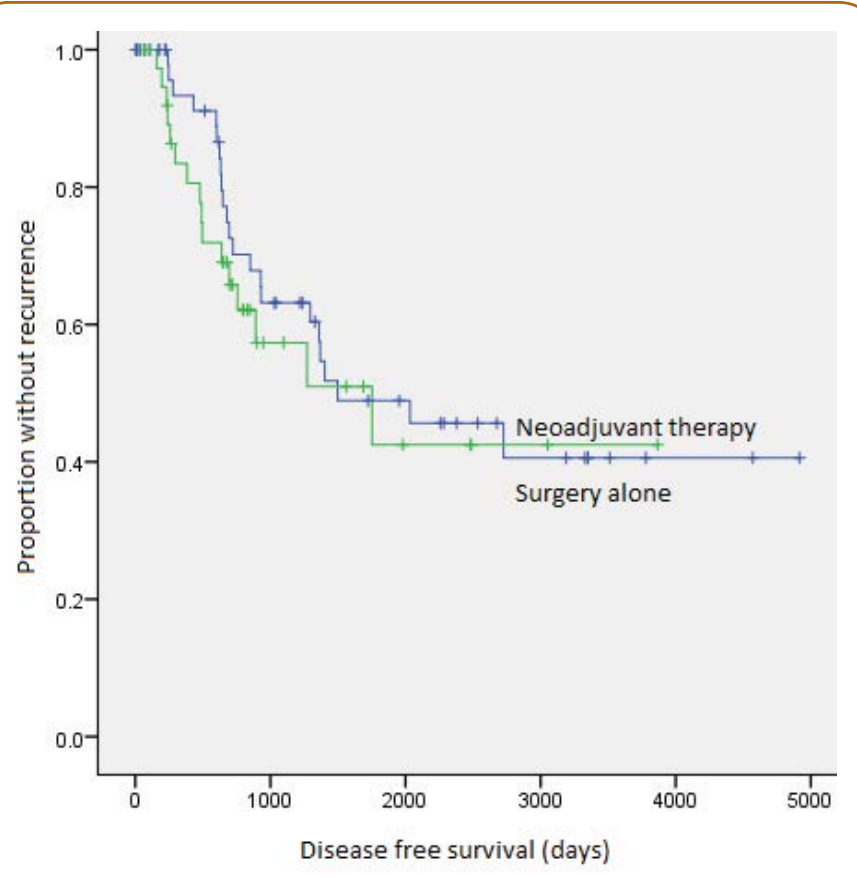

Figure 1 Kaplan-Meier plot for disease recurrence $(P=0.38)$.

Disease free survival, expressed as the proportion of patients without recurrence postoperatively, revealed no significant difference between both patient groups (P-value 0.38) (Figure 1). The median disease free survival for the neoadjuvant therapy group was 58.4 months $(\mathrm{Cl} 15.3,101.4)$, compared to 49 months $(\mathrm{Cl} 7.9,92.0)$ for the surgery alone group. The overall median disease free survival was 49.2 months ( $\mathrm{Cl} 39.1,68.3$ ).

\section{Discussion}

Previously, oesophagectomy or definitive chemoradiotherapy were the only treatment options used in the management of localised oesophageal cáncer [11]. In recent years, multimodality treatment strategies involving neoadjuvant chemotherapy and radiotherapy followed by surgery have become more widespread, as these have been shown to improve disease free survival [12]. The impact of neoadjuvant therapy on perioperative morbidity and mortality has been uncertain $[12,13]$. This study revealed a decrease in adverse events after the introduction of neoadjuvant therapy in a low volume hospital. When considering perioperative mortality, there were three deaths in the surgery alone group, compared to no deaths in the neoadjuvant therapy group. There was no increase in perioperative complications as a consequence of neoadjuvant therapy. A recent meta-analysis found higher rates of complete resection among patients who underwent neoadjuvant therapy [14]. This study had a similar finding, with a higher percentage of complete resection among patients treated with neoadjuvant therapy, although this difference did not reach statistical significance. This finding suggests that neoadjuvant therapy may be beneficial in practice, by the facilitation of tumour downstaging [6]. Alternatively, neoadjuvant therapy may have resulted in a degree of patient selection due to the possible deterioration of the patient's condition during their preoperative period.

With regard to disease free survival, this study was unable to detect a significant difference in recurrence rates between the two patient groups. The randomised controlled trial performed by the CROSS group revealed improved rates of both overall and disease-free survival among patients who received neoadjuvant chemoradiotherapy followed by surgery [14]. Other studies have suggested that neoadjuvant therapy may play a role in reducing disease recurrence, by facilitating complete resection and targeting micrometastases [6].

This study has various limitations. As a retrospective review, the nonrandomised allocation of patients may have led to selection bias. Further, patients were less likely to undergo neoadjuvant therapy in the first half of the study period (2001-2006) when compared to the second half of the study period (2007-2012). The decrease in morbidity and mortality in the neoadjuvant group may have resulted from improvement in perioperative care in the later years of the study.

\section{Conclusion}

Overall, this study revealed that the administration of neoadjuvant therapy to patients with cancer of the oesophagus or gastro-oesophageal junction, at the Canberra Hospital between 2001 and 2012, was associated with a decrease in the rate of perioperative complications. Furthermore, neoadjuvant therapy may be associated with improved rates of complete resection. Unfortunately, in our small group of patients, we were unable to detect an improved disease free survival in the neoadjuvant therapy group.

\section{Acknowledgement}

The authors would also like to thank Gloria Spyropoulos, Research and QA Manager, Medical Records, Canberra Hospital and Teresa Neeman, Statistical Consultant at the Australian National University for their contributions to this study. 


\section{References}

1 Lv J, Cao X, Zhu B, Ji L, Tao L, et al. (2009) Effect of neoadjuvant chemoradiotherapy on prognosis and surgery for esophageal carcinoma. World J. Gastroenterol 15: 4962-4968.

2 Ben-David K, Rossidis G, Zlotecki R, Grobmyer SR, Cendan JC (2011) Minimally invasive esophagectomy is safe and effective following neoadjuvant chemoradiation therapy. Ann Surg Oncol 18: 33243329.

3 Besharat S, Jabbari A, Semnani S, Keshtkar A Marjani J, et al. (2008) Inoperable esophageal cancer and outcome of palliative care. World J Gastroenterol 14: 3725-3728.

4 Kato H, Fukuchi M, Manda R, Faried A, Takita J, et al. (2004) The effectiveness of planned esophagectomy after neoadjuvant chemoradiotherapy for advanced esophageal carcinomas. Anticancer Res 24: 4091-4096.

5 Stewart J, Hoff S, Johnson D, Murray MJ, Butler DR, et al.(1993) improved survival with neoadjuvant therapy and resection for adenocarcinoma of the esophagus. Ann Surg 218: 571-578.

6 Urschel J, Vasan H, Blewett C (2002) A meta-analysis of randomized controlled trials that compared neoadjuvant chemotherapy and surgery to surgery alone for resectable esophageal cancer. Am J Surg 183: 274-299.

7 Sutton P, Clark P (2000) Neo-adjuvant treatment for oesophageal cancer. GI Cancer 3: 231-238.
8 Urschel J, Vasan H (2003) A meta-analysis of randomized controlled trials that compared neoadjuvant chemoradiation and surgery to surgery alone for resectable esophageal cancer. Am J Surg 185: 538-543.

9 Van Hagen P, Hulshof M, van Lanschot J, Steyerberg E.W, van Berge Henegouwen MI, et al. (2012) Preoperative chemoradiotherapy for esophageal or junctional cancer. N Engl J Med. 2012; 366: 2074-2084.

10 Hagry O, Coosemans W, De Leyn P, Nafteux P, Van Raemdonck D, et al. (2003) Effects of preoperative chemoradiotherapy on postsurgical morbidity and mortality in T3-4 +/- Ml cancer of the oesophagus and gastro-oesophageal junction. Eur J Cardiothorac Surg 24: 179-786.

11 Dindo D, Demartines N, Clavien P (2004) Classification of surgical complications. Ann Surg 244: 931-937.

12 Melis M, McLoughlin J, Dean E, Siegel EM, Weber JM, et al. (2009) Correlations between neoadjuvant treatment, anemia, and perioperative complications in patients undergoing esophagectomy for cancer. J Surg Res 153: 114-120.

13 Bosset J, Gignoux M, Triboulet J (1997) Chemoradiotherapy followed by surgery compared with surgery alone in squamous cell cancer of the esophagus. N Engl J Med 667: 161-167.

14 Kranzfelder M, Schuster T, Geinitz H, Friess H, Buchler P (2011) Metaanalysis of neoadjuvant treatment modalities and definitive nonsurgical therapy for oesophageal squamous cell cancer. Brit J Surg 98: 768-783. 